Case Report

\title{
An Uncommon Twist: Isolated Fallopian Tube Torsion in an Adolescent
}

\author{
Sundeep Kisku and Reju Joseph Thomas \\ Department of Paediatric Surgery, Christian Medical College, Vellore, Tamil Nadu 632004, India
}

Correspondence should be addressed to Sundeep Kisku; skisku@yahoo.com

Received 13 June 2013; Accepted 22 July 2013

Academic Editors: D. J. Bentrem, A. Darwish, and A. R. Novotny

Copyright ( $) 2013$ S. Kisku and R. J. Thomas. This is an open access article distributed under the Creative Commons Attribution License, which permits unrestricted use, distribution, and reproduction in any medium, provided the original work is properly cited.

We report a 13-year-old girl with bilateral paratubal cysts and left isolated fallopian tube torsion (IFTT). Paratubal cysts are uncommon in children, and IFTT is a rare complication. Awareness of this entity and prompt surgical intervention could potentially salvage the fallopian tube preserving fertility.

\section{Introduction}

Paratubal cysts occur in about $4 \%$ of adolescents [1] and can rarely undergo torsion along with the adnexal structures. Isolated fallopian tube torsion (IFTT) occurs when the fallopian tube torses without ovarian torsion. The incidence is 1 in 1500000 women [2]. Delay in diagnosis and treatment occur due to lack of definitive clinical features. Awareness of this entity, early diagnosis, and prompt treatment can salvage the torted fallopian tube and preserve fertility.

\section{Case History}

A 13-year-old postpubertal girl presented with vomiting, left flank, and lower abdominal pain for 5 days. She reported a similar, albeit milder episode 3 months ago. She was being treated by the emergency department for suspected urinary tract infection. On examination, she was afebrile and hemodynamically stable. There was tenderness in the left iliac fossa and differential tenderness on rectal examination, in the Pouch of Douglas.

Her hemoglobin was $11.0 \mathrm{gm} \%$ and total leukocyte count 10,600 cells/cc ( $66 \%$ polymorphs, $34 \%$ lymphocytes). Urine microscopy revealed RBC 15-20 cells/high power field (HPF) and WBC 6-8 cells/HPF. Abdominal sonography revealed a right ovarian cyst $6.4 \times 3.8 \times 5.4 \mathrm{~cm}$.

With a provisional diagnosis of torsion of the ovary, emergency laparoscopy was performed. The distal third of the left fallopian tube along with a $5 \times 5 \mathrm{~cm}$ paratubal cyst (Figures 1 and 2) had undergone gangrenous torsion (2.5 turns). Both ovaries were normal. The right fallopian tube had several paratubal cysts in its distal third. The torted left cyst contained $40 \mathrm{~mL}$ of hemorrhagic fluid which was aspirated. The nonviable left fallopian tube and cyst were detorted and excised. The right paratubal cysts (three) were punctured, and clear fluid was drained. Histopathology revealed the left fallopian tube and cyst to be extensively congested, hemorrhagic, and infarcted.

The postoperative period was uneventful, and she was discharged two days later. The parents were counseled of possible infertility in the future due to fallopian tubal stenosis secondary to the paratubal cysts.

\section{Discussion}

IFTT occurs mostly in 12-15 year olds and is rare before menarche or during menopause. IFTT may occur due to intrinsic and extrinsic causes [3]. Intrinsic causes include congenital anomalies (long or spiral tube) acquired pathology (hydrosalpinx, cysts, pelvic inflammatory disease) and autonomic dysfunction with abnormal peristalsis. Extrinsic causes include local factors and mechanical factors, jarring movements of the body (Sellheim theory).

Paratubal cysts arise from Mullerian or Wolffian structures and are common in adult females (92\% of hysterectomies). These are hormone sensitive and are generally 


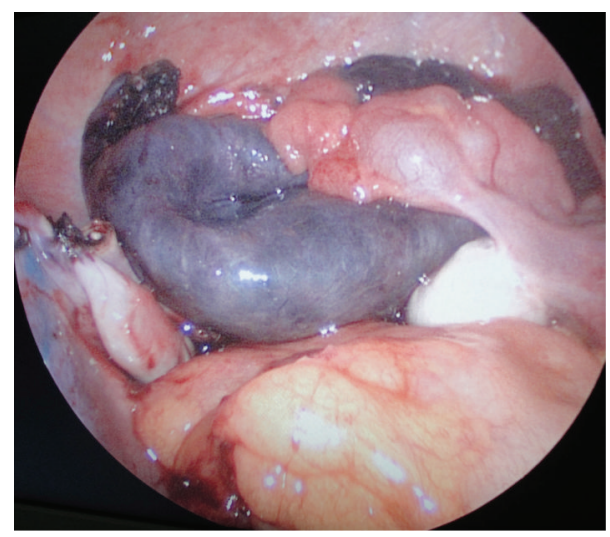

FIGURE 1: Normal ovaries with gangrene of the distal third of the left fallopian tube and paratubal cyst (postdetorsion and excision view). The right fallopian tube is seen with a few small paratubal cysts.

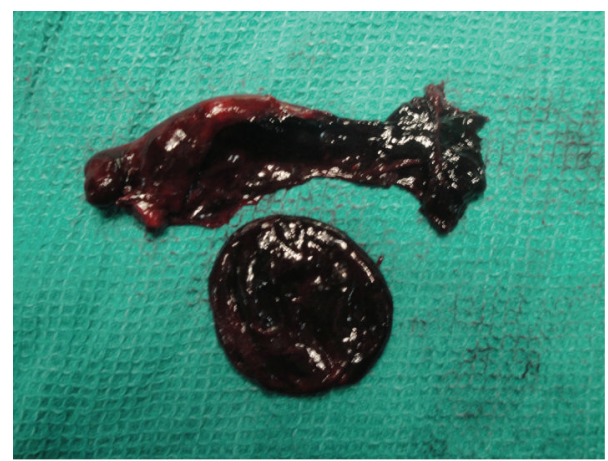

FIgURE 2: Left fallopian tube with paratubal cyst.

asymptomatic [1]. However, these are uncommon in children. Hemorrhage, rupture, and infertility are rare complications and occur in large cysts. IFTT has been reported in with unilateral [4] and bilateral [5] paratubal cysts in older children. Small cysts $(<3 \mathrm{~cm})$ may be punctured and larger ones excised. Malignant neoplasms arising from paratubal cysts are very rare [6]. Regular followup with abdominal sonogram is advisable.

The most common symptom is ipsilateral lower abdominal or flank pain. Physical findings include abdominal tenderness with or without peritoneal signs and adnexal tenderness on rectal examination. A mass may not be palpable. Laboratory values are usually nonspecific.

The sonographic findings include normal appearing ovaries, free fluid, a dilated tube with thickened, echogenic walls, and internal debris representing a torsed tube [7]. A whirlpool sign specific of IFTT has been described [8]. Paratubal cysts are difficult to diagnose preoperatively, and even transvaginal ultrasound in older women has detected only about $44 \%$ of paratubal cysts preoperatively [6]. Doppler study may show high impedance or absence of flow in a tubular structure. CT findings of IFTT include an adnexal cyst separate from the ovary, a twisted appearance to the fallopian tube, dilated tube greater than $15 \mathrm{~mm}$, a thickened and enhancing tubal wall, and luminal CT attenuation greater than $50 \mathrm{H}$ consistent with hemorrhage [9]. MRI abdomen is informative in complex situations when the ultrasound is indeterminate.

Laparoscopy is the gold standard in the diagnosis and management of paratubal cysts complicated with IFTT. Adnexal detorsion with resection of the paratubal cyst and preservation of the fallopian tube (if not gangrenous) is the procedure of choice. Fixation of the detorted tube is controversial. Surgery is usually done too late for tubal conservation. A few reports of detorsion salvaging the fallopian tube with subsequent pregnancy have been reported [10]. Salpingostomy, though unpopular for its potential to cause ectopic pregnancies, has been speculated to improve fertility, as a second stage management following salpingectomy [11].

\section{Key Messages}

Paratubal cyst causing isolated fallopian tube torsion is an uncommon cause of pain in an adolescent. Awareness and prompt treatment can potentially salvage the fallopian tube preserving fertility.

\section{References}

[1] M. Samaha and J. D. Woodruff, "Paratubal cysts: frequency, histogenesis, and associated clinical features," Obstetrics and Gynecology, vol. 65, no. 5, pp. 691-694, 1985.

[2] O. H. Hansen, "Isolated torsion of the fallopian tube," Acta Obstetricia et Gynecologica Scandinavica, vol. 49, no. 1, pp. 36, 1970.

[3] A. F. Youssef, M. M. Fayad, and M. A. Shafeek, "Torsion of the fallopian tube. A clinico-pathological study," Acta Obstetricia et Gynecologica Scandinavica, vol. 41, pp. 292-309, 1962.

[4] R. K. Bloom and S. Larusso, "Paratubal cyst with isolated torsion of the fallopian tube," Journal of Diagnostic Medical Sonography, vol. 25, no. 1, pp. 58-61, 2009.

[5] S. Seshadri, A. Morris, D. Uchil, and A. Joloaso, "Bilateral paratubal cysts with co-existent fallopian tube torsion in an adolescent," Journal of Obstetrics and Gynaecology, vol. 29, no. 6, pp. 564-565, 2009.

[6] A. M. Darwish, A. F. Amin, and S. A. Mohammad, "Laparoscopic management of paratubal and paraovarian cysts," Journal of the Society of Laparoendoscopic Surgeons, vol. 7, no. 2, pp. 101106, 2003.

[7] M. Gross, S. L. Blumstein, and L. C. Chow, "Isolated fallopian tube torsion: a rare twist on a common theme," The American Journal of Roentgenology, vol. 185, no. 6, pp. 1590-1592, 2005.

[8] S. B. Vijayaraghavan and S. Senthil, "Isolated torsion of the fallopian tube: the sonographic whirlpool sign," Journal of Ultrasound in Medicine, vol. 28, no. 5, pp. 657-662, 2009.

[9] M. A. Ghossain, J. N. Buy, M. Bazot et al., "CT in adnexal torsion with emphasis on tubal findings: correlation with US," Journal of Computer Assisted Tomography, vol. 18, no. 4, pp. 619-625, 1994.

[10] C. R. Blair, “Torsion of the fallopian tube," Surgery, Gynecology and Obstetrics, vol. 114, pp. 727-730, 1962.

[11] S. A. Boukaidi, J. Delotte, H. Steyaert et al., "Thirteen cases of isolated tubal torsions associated with hydrosalpinx in children and adolescents, proposal for conservative management: retrospective review and literature survey," Journal of Pediatric Surgery, vol. 46, no. 7, pp. 1425-1431, 2011. 


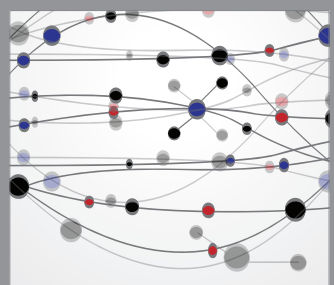

The Scientific World Journal
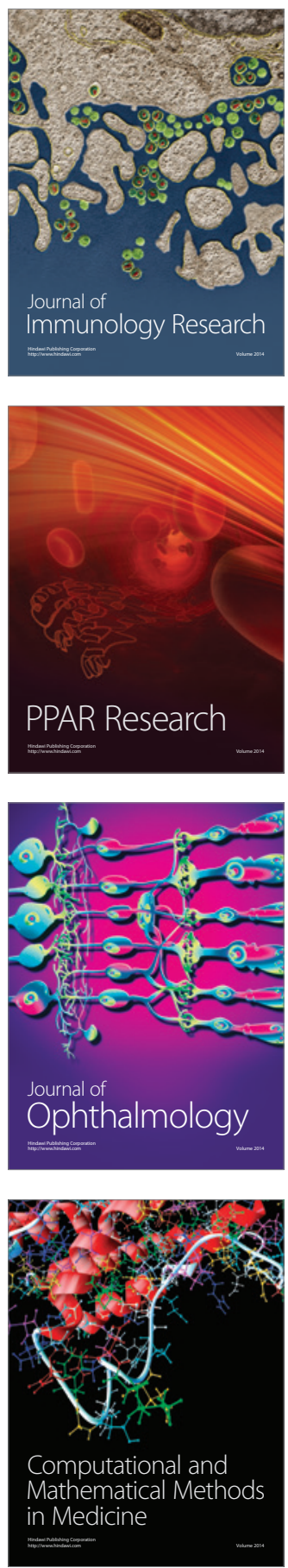

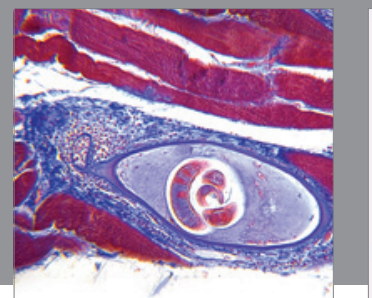

Gastroenterology

Research and Practice
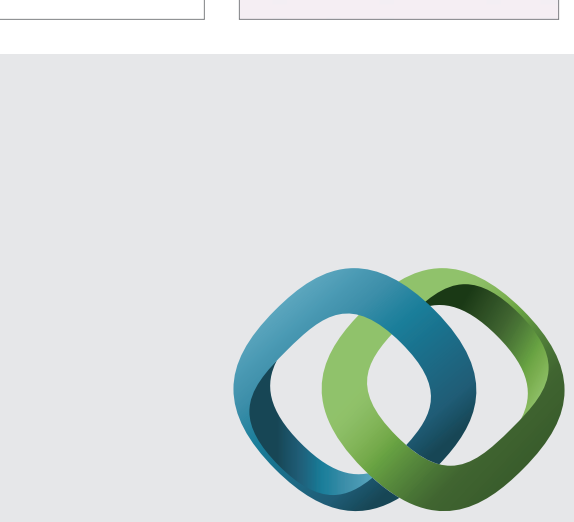

\section{Hindawi}

Submit your manuscripts at

http://www.hindawi.com
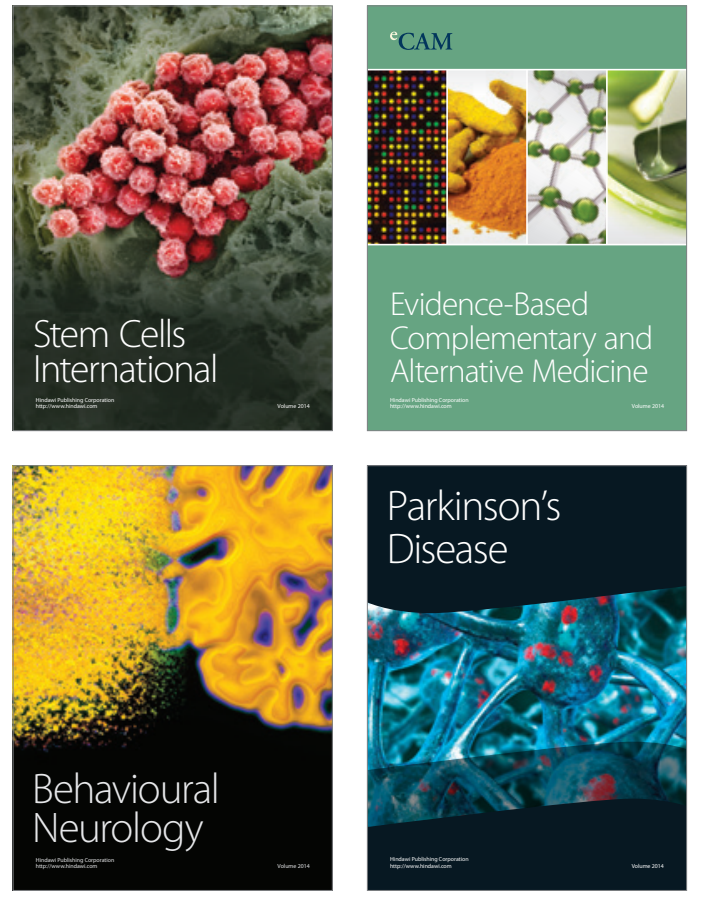
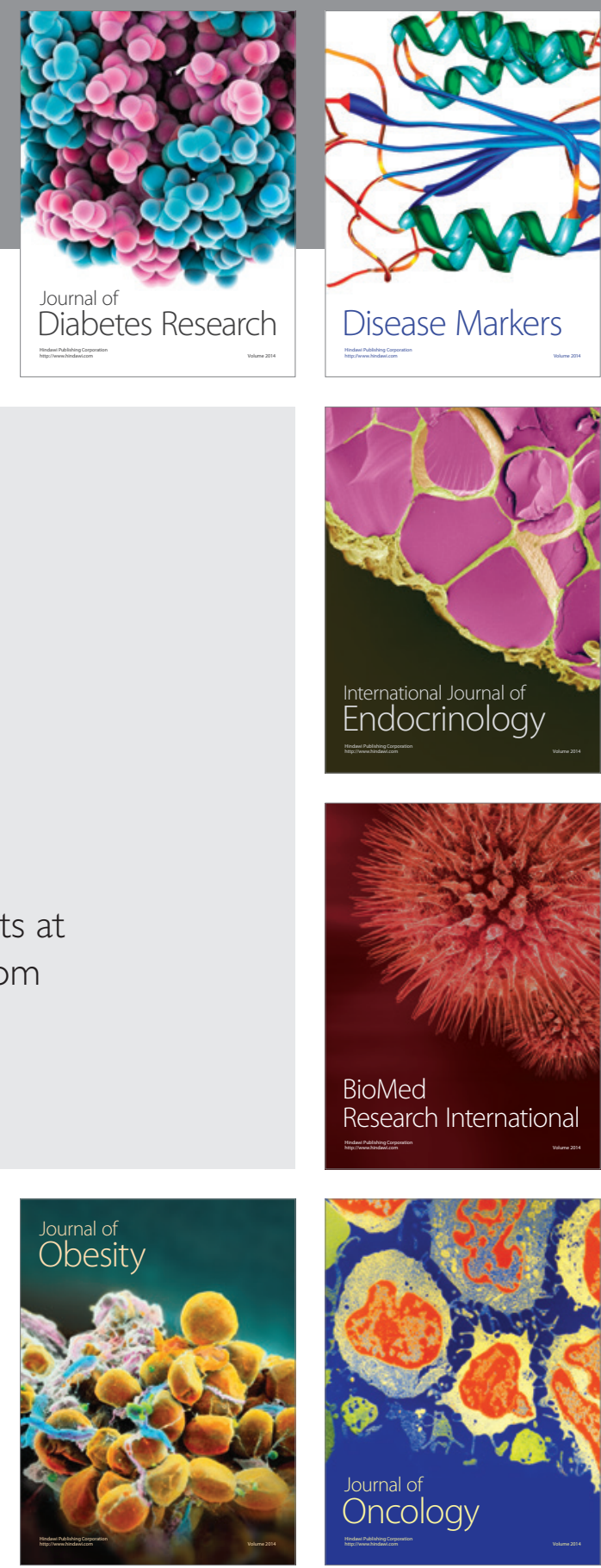

Disease Markers
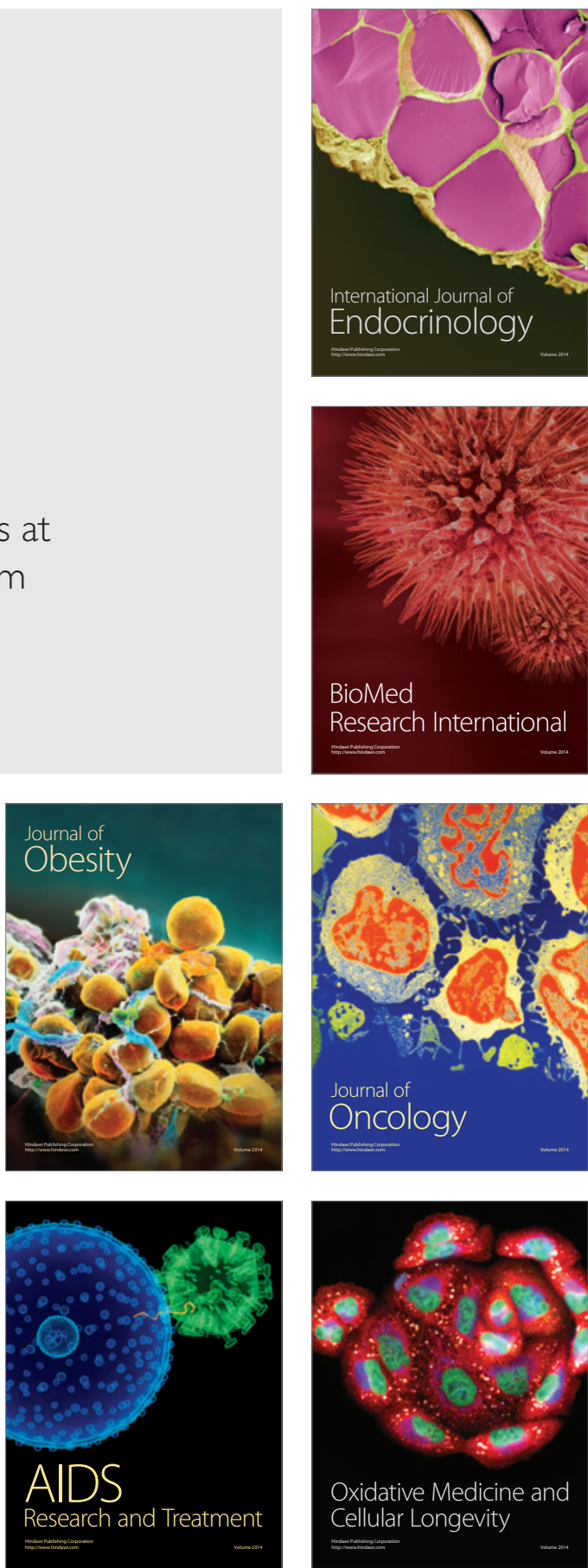